\title{
Pudendal schwannoma: A case report and literature review
}

\author{
Clarisse R. Mazzola, MD; Nicholas Power, MD; Mark H. Bilsky, MD; Roger Robert, MD; ${ }^{\dagger}$ \\ Bertrand Guillonneau, $M D^{*}$
}

*Urology Service, Surgery Department, Memorial Sloan-Kettering Cancer Center, New York, NY; †Department of Neurosurgery, Memorial Sloan-Kettering Cancer Center, New York, NY; §Neurotraumatology Department, L'Hôtel-Dieu de Nantes, Centre Hospitalier Universitaire, Nantes, France

Cite as: Can Urol Assoc J 2014;8(3-4):e199-203. http://dx.doi.org/10.5489/cuaj.1734 Published online March 11, 2014.

\section{Abstract}

Schwannomas are benign nerve sheath tumours most often associated with the cranial nerves and the peripheral nerve system of the neck and extremities. Pelvic schwannomas are rare, with only about 25 cases reported. We report the case of a 34-year-old man referred for worsening pain of 10 years duration involving the right testicle and right penile shaft. Magnetic resonance imaging discovered a well-circumscribed pelvic tumour of $3.2 \times 2.8 \times 3.2 \mathrm{~cm}$. Considering the possible complications involved in exposing the pudendal nerve during surgical resection, we performed an extensive literature search to aid preoperative planning. The most commonly described surgical approach for pelvic schwannomas has been open median laparotomy with transperitoneal dissection. To our knowledge, pudendal schwannomas have never been described in the literature. However, after considering the location and characteristics of the tumour, we chose laparoscopy because it offers the advantages of better visualization of anatomical structures with minimal invasiveness and faster recovery. At the 3-week follow-up, the patient described a significant decrease in pain and normal neurological and urological examinations.

\section{Introduction}

Schwannomas are benign nerve sheath tumours composed of Schwann cells. They comprise $5 \%$ of all benign soft tissue tumours. ${ }^{1}$ They have a propensity for the cranial nerves and the peripheral nervous system of the neck, mediastinum and extremities. Benign retroperitoneal schwannomas are rare, and among those, pelvic schwannomas are particularly unusual. There are 25 reported cases. ${ }^{2}$ Their deep location in an anatomically complex region raises issues concerning the strategy of surgical approach for resection. To date the most commonly described surgical approach for pelvic schwannomas has been open median laparotomy with transperi- toneal dissection. ${ }^{2}$ Only 2 cases of laparoscopy have been reported; ${ }^{3,4}$ other approaches, such as transischiorectal, ${ }^{5}$ transperineal, ${ }^{6}$ transgluteal $^{7}$ and transvaginal, ${ }^{8}$ are just as rare.

We report a case of a pudendal schwannoma removed laparoscopically. We also discuss other potential surgical approaches.

\section{Case report}

A 34-year-old male was referred in 2010 for worsening rightsided perineal pain and a 10-year history of fasciculations. Presumed diagnosis was prostatitis and he was treated with multiple courses of fluoroquinolone antibiotics, but failed to improve. The other predominant symptoms were paresthesias involving the right testicle and right penile shaft. The symptoms were determined to be related to a pelvic mass discovered on radiological workup. His medical history revealed no significant illnesses. He had undergone previous surgery for excision of a pilonidal cyst, laparoscopic bilateral hernia repair in 2007, and penile shaft skin tag removal in 2008. He had no history of malignancies and an unremarkable family history. On physical examination, there was no evidence of cutaneous lesions or palpable lymphadenopathy and his abdomen was soft, non-tender and without any palpable masses. The International Prostate Symptom Score (IPPS) and Sexual Health Inventory for Men (SHIM) score were 0 and 24, respectively. The neurological examination highlighted a hypoesthesia of the right penile shaft. The rest of the neurological exam was normal. A right-sided soft mass was palpable on digital rectal examination (DRE).

Magnetic resonance imaging (MRI) of the abdomen and pelvis revealed an extraperitoneal $3.2 \times 2.8 \times 3.2-\mathrm{cm}$ soft tissue mass along the right posterior pelvic side wall above the muscular pelvic floor. The sacral plexus branches were seen coursing superiorly and anterolateral to the mass as they exited through the sciatic notch. Lateral sacral branches also coursed posterior to the mass. The ureter was identified anteriorly. There was heterogeneous enhancement (Fig. 1). 


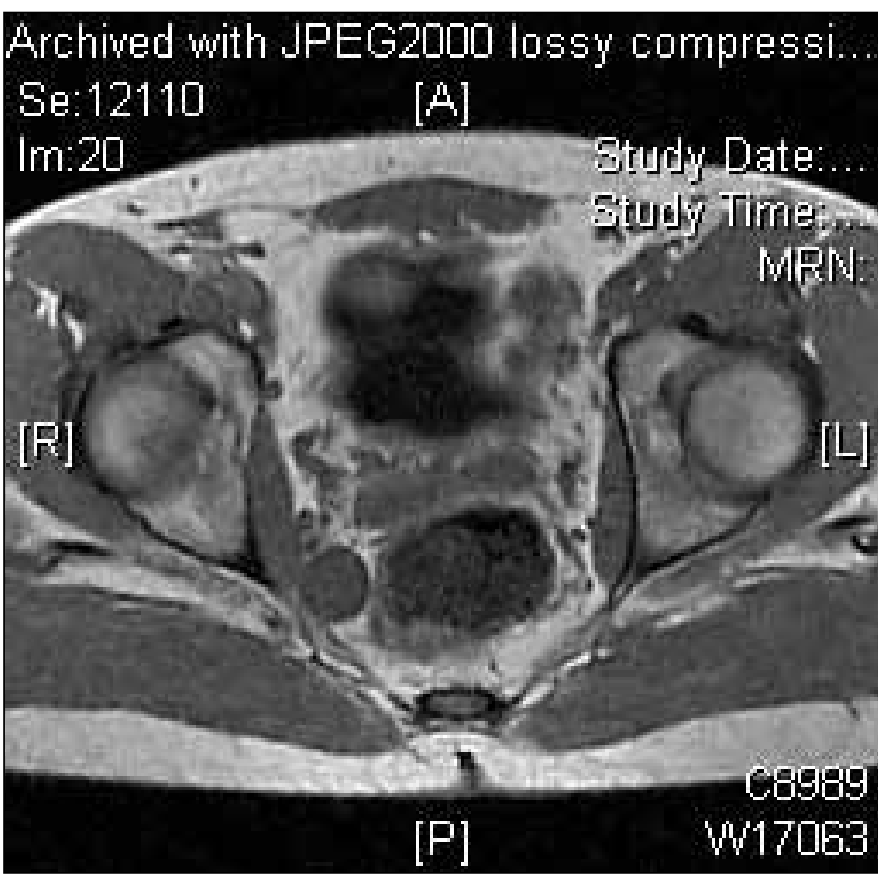

Fig. 1. A magnetic resonance image of the abdomen and pelvis shows the extraperitoneal $3.2 \times 2.8 \times 3.2$-cm well-circumscribed soft tissue mass along the right posterior pelvic sidewall above the muscular pelvic floor.

Subsequent comparison to a previous MRI, performed in 2005 for an unrelated spinal complaint and not commented on at that time, revealed that the lesion measured $2 \mathrm{~cm}$. The interval growth for this pelvic nerve mass was $1.2 \mathrm{~cm}$ in 5 years.

At the patient's request, and with his informed consent, a laparoscopic resection of the pelvic mass was performed. Prior to resection, the patient was referred for sperm banking. After the patient was given general anaesthesia and placed in the supine position, a DRE identified a mobile, well-circumscribed tumour along the right aspect of the rectum. Continuous intraoperative electromyographic (EMG) monitoring was performed.

Three ports were initially placed: one $12 \mathrm{~mm}$ at the umbilicus and two $5 \mathrm{~mm}$ working ports on either side of the patient's umbilicus triangulating down into the right pelvis. The dissection was performed along the right iliac fossa, incising the peritoneum overlying the right umbilical ligament. The dissection was then carried down to the level of the endopelvic fascia along the hypogastric artery and its lateral branches. A third 5-mm port was then inserted in the midline below the umbilicus for retraction of the umbilical ligament. After incision of the right endopelvic fascia, the tumour was identified lateral and posterior to the prostatic pedicle. Posterior dissection with cold scissors was then performed, sparing the neurovascular structures along the prostate, rectum and right pelvic side wall. The thin capsule overlying the mass was incised and the tumour was dissected circumferentially, leaving the posterior capsule (Fig. 2). The

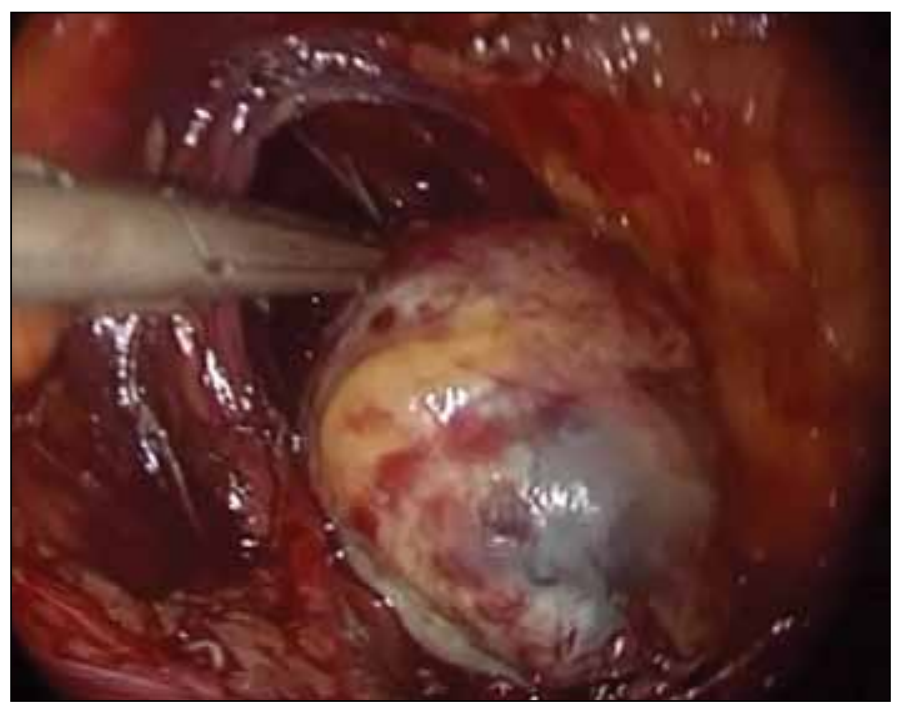

Fig. 2. Laparoscopic view of the pudendal schwannoma dissection.

tumour was dissected free with no complications. Bipolar electrocautery was used for meticulous hemostasis. The pelvic tumour was then extracted from the abdomen through a slight extension of the umbilical port site. Intraoperative frozen section analysis showed that the tumour was consistent with a nerve schwanomma. The postoperative course was uneventful and the patient was discharged from the hospital the next day.

Of note, intraoperative nerve stimulation monitoring identified that the schwannoma arose from the pudendal nerve adjacent to the pelvic plexus. However, stimulation after the resection confirmed a normal muscular anal response.

Final pathology revealed a $11.5 \mathrm{~g}, 3 \times 3 \times 2.2$-cm ovoid, red-pink to tan-grey portion of soft tissue consistent with a schwannoma. Sectioning revealed tan-yellow, whorled, diffusely hemorrhagic, rubbery cut surfaces with a thin, fibrous capsule (Fig. 3).

At the neurosurgery and urology follow-up visits 3 weeks later, the patient described significant improvement of his perineal pain and resolution of the intermittent fasciculations. The neurological and urological examinations were normal. SHIM and IPSS scores had not changed since preoperative testing.

\section{Discussion}

The pudendal nerve arises from sacral nerves S2-S4 and, after passing between the piriformis and coccygeus muscles, leaves the pelvis through the distal part of the greater sciatic foramen. This nerve accompanies the pudendal vessels along the lateral wall of the ischiorectal fossa within Alcock's canal. This canal, also called the pudendal canal, is formed by the obturator internus fascia. Along its course, the pudendal nerve gives rise to the inferior rectal nerve, the 


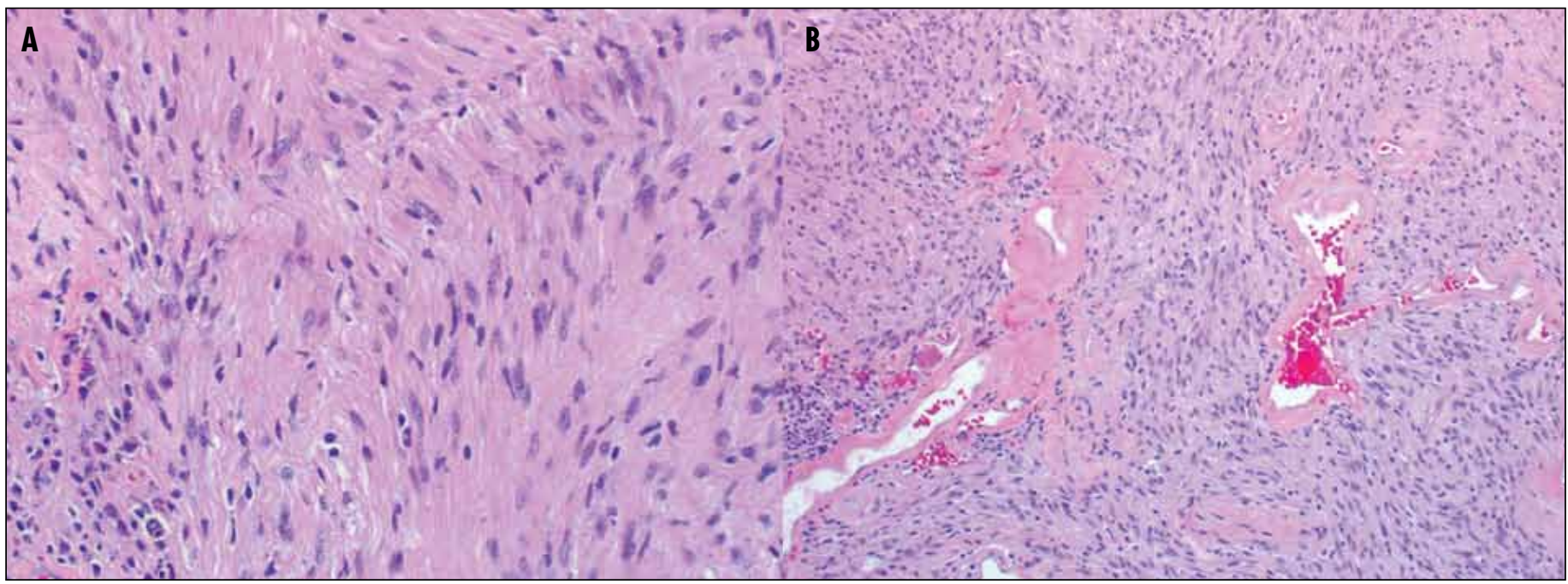

Fig. 3. Photomicrograph (original magnification, $\times 200$; hematoxylin-eosin stain) reveals that the tumour consists of focal cellular (Antoni A) areas and hypocellular (Antoni B) areas with vacuolar degeneration.

perineal nerve, and the dorsal nerve to the penis or clitoris. Accordingly, it supplies the somatic anal and urethral sphincters, as well as penile and clitoral musculature. ${ }^{5,7}$

Functionally, the pudendal nerve ensures the sensitivity of the perineum teguments (the glans penis, clitoris, scrotum, labia majora, skin of the central fibrous perineal body, and anus). It also ensures the motor innervation of the erector muscles and the striated sphincters, thus controlling erection and the voluntary actions of the sphincters. It is mostly involved in sexual activity, micturition and defecation. ${ }^{7}$

Most schwannomas are benign nerve sheath tumours. They are well-circumscribed and do not invade local tissues, making them amenable to dissection. ${ }^{2}$ The primary treatment is surgical excision. Most authors recommend complete excision with preservation of the associated nerve when identified. Recurrence has been associated only with incomplete resection. To our knowledge, schwannomas of the pudendal nerve have never been described in the literature. Considering the difficulty and possible functional complications involved in exposing the pudendal canal and nerve while attempting to cure this benign lesion, we performed an extensive literature search to aid preoperative planning of the surgical approach (Fig. 4).

Open median laparotomy with a transperitoneal dissection is by far the most commonly reported approach. ${ }^{2}$ It is associated with the usual risks of a laparotomy, including perioperative intestinal injury, postoperative peritonitis, wound infection and peritoneal adhesions. Some cases of sexual functional impairment after transperitoneal dissection to the retroperitoneum have also been reported. ${ }^{2}$

A recently described alternative open technique consists of a transgluteal approach, which allows access to the pudendal nerve between the sacrospinous and the sacrotuberous ligaments (Fig. 5). ${ }^{7}$
Another option is the transperineal approach. The ischiorectal fossa is opened through a vertical incision of the skin between the anus and the ischial tuberosity. The inferior rectal nerve is then traced to the entrance of Alcock's canal. ${ }^{6}$ In a large series of 212 patients, Bautrant and colleagues described a transischiorectal approach to the pudendal nerve, ${ }^{5}$ mostly for pudendal decompression (104 cases). The use of a transvaginal approach in a female patient has also been reported. ${ }^{8}$

The more recent alternative option is laparoscopy. This technique is a very well-described approach to the upper retroperitoneal space. It has been reported in 4 cases of upper retroperitoneal schwannomas, ${ }^{9}$ with a transabdominal approach in 3 of these cases. In the fourth case, Nishi and colleagues reported the endoscopic resection of a pelvic neurogenic tumour via the retroperitoneal approach. ${ }^{9}$

As the presacral-retrorectal space communicates superiorly with the retroperitoneal space at the level of the peritoneal reflection (S2-S3 vertebrae), it is considered its downward extension..$^{3,4,7}$ The laparoscopic approach to this area with the intention to treat a pelvic schwannoma has been described twice, ${ }^{3,4}$ but never for pudendal schwannomas. The first time was in 1996 by Melvin. ${ }^{3}$ The second time was in 2005 by Rousseau and colleagues for a benign schwannoma of the sacral plexus. ${ }^{4}$ Laparoscopy theoretically offers several advantages over traditional surgical techniques in the resection of pudendal schwannomas. The specific risks of open surgery are avoided, and the magnified view of the tumour and its surrounding anatomic features potentially enables a better and safer dissection. Recovery is typically faster, with a shorter hospital stay, and the cosmetic outcomes are also more favourable.

In our case, it is possible that open resection would have been performed if there had been a high suspicion 


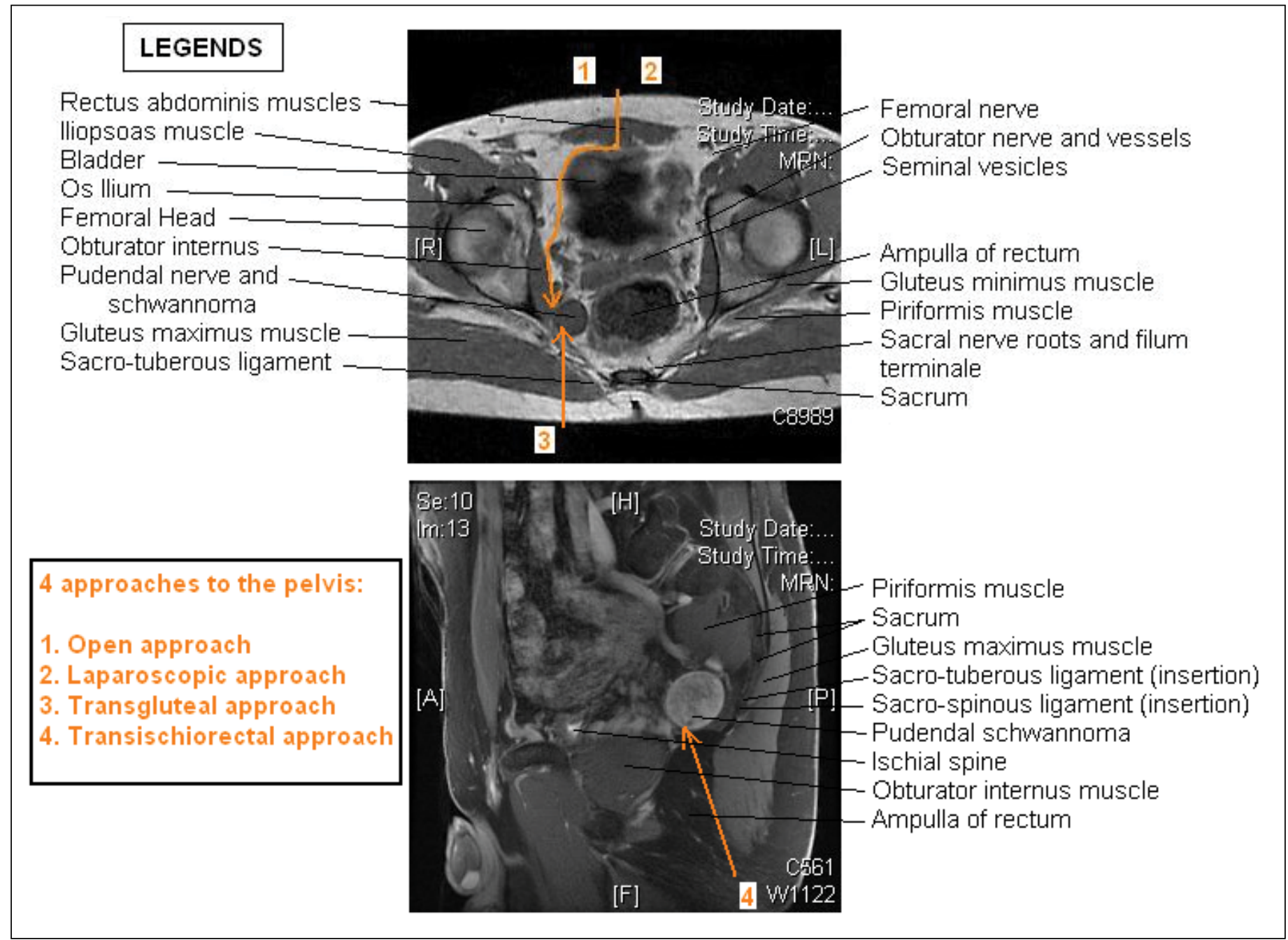

Fig. 4. A magnetic resonance imaging cross-section cut showing the different possible surgical approaches to the pelvis to treat our tumour.

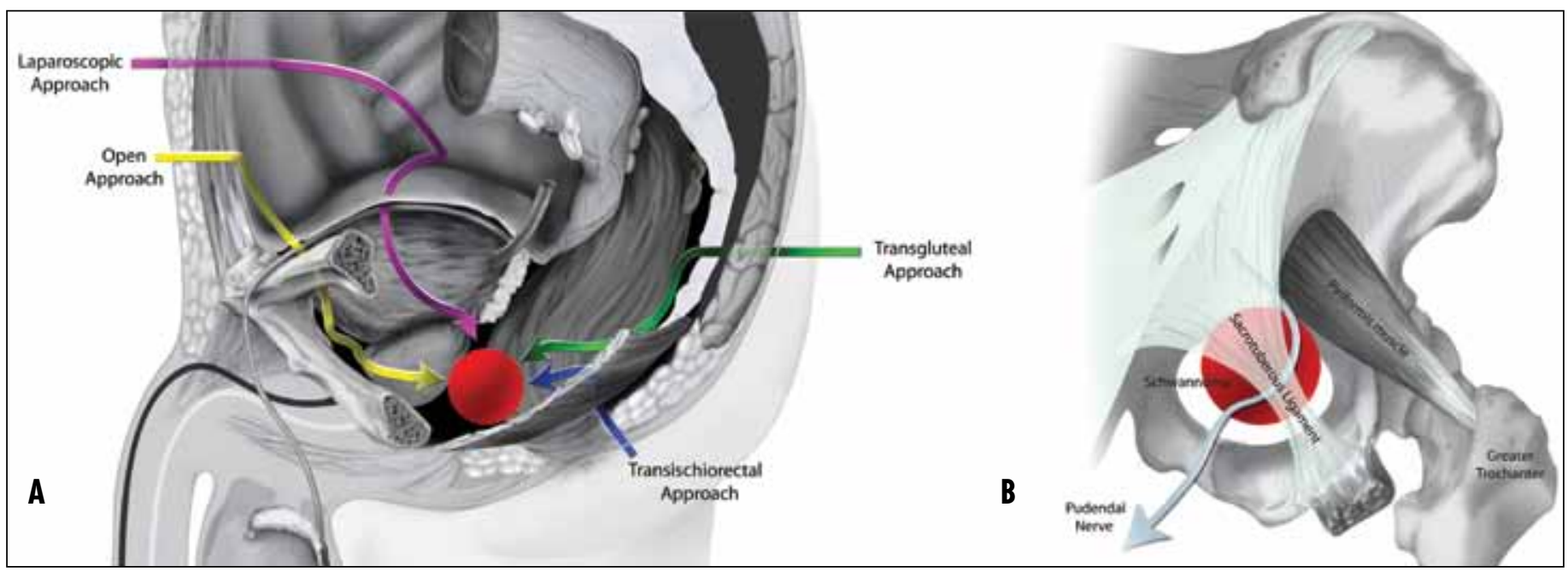

Fig. 5. Possible surgical approaches to the pelvis. 
of malignancy. In this location, $90 \%$ of malignancies are retroperitoneal sarcomas and about $10 \%$ are extragonadal germ cell tumours. ${ }^{10}$ But the appearance of a well-circumscribed, encapsulated, non-locally invasive, slow-growing tumour with no regional lymphadenopathy allowed us to confidently differentiate it from the malignant neoplasms.

Indeed, a retrospective study of 25 cases of retroperitoneal tumours found a significant correlation between irregular margins and malignancy. ${ }^{11}$ The authors developed a retroperitoneal tumour scoring system to distinguish primary retroperitoneal benign tumours from their malignant counterparts based on 5 criteria: (1) maximum diameter equal to or larger than $5.5 \mathrm{~cm}$, (2) presence of symptoms, (3) absence of calcification, (4) presence of irregular margins and (5) presence of cystic degeneration or necrosis. They found a significant correlation between the incidence of malignant tumours and the total retroperitoneal tumour score.

For our patient, the final diagnosis was a pudendal schwanomma. Other benign tumours that could have been suspected include retroperitoneal pseudocyst, abscess and lymphocele, in which case the tumour would have been almost entirely cystic, which is rarely the case for schwannomas $^{12}$ and was not the case here. If the tumour had been mostly solid, or if there was associated lymphadenopathy, we would have considered neurofibroma and lymphoma as possible differential diagnoses.

\section{Conclusion}

Laparoscopy is a possible approach for benign pelvic tumours. It potentially offers the advantages of better visualization of anatomical structures with minimal invasiveness and faster postoperative recovery.
Competing interests: Dr. Mazzola, Dr. Power, Dr. Bilsky, Dr. Robert and Dr. Guillonneau all declare no competing financial or personal interests.

This paper has been peer-reviewed.

\section{References}

1. Kransdorf MJ. Benign soft-tissue tumors in a large referral population: distribution of specific diagnoses by age, sex, and location. AJR Am J Roentgenol 1995;164:395-402. http://dx.doi.org/10.2214/air.164.2.7839977

2. Hughes MJ, Thomas JM, Fisher $C$, et al. Imaging features of retroperitoneal and pelvic schwannomas. Clin Radiol 2005;60:886-93. http://dx.doi.org/10.1016/i.crad.2005.01.016

3. Melvin WS. Laparoscopic resection of a pelvic schwannoma. Surg Laparosc Endosc 1996;6:489-91. http://dx.doi.org/10.1097/00019509-199612000-00015

4. Rousseau MA, Pascal-Mousselard $\mathrm{H}$, Lazennec JY, et al. The mini-invasive anterior extra peritoneal approach to the pelvis. Eur J Surg Oncol 2005;31:924-6. http://dx.doi.org/10.1016/i.eiso.2005.05.006

5. Bautrant $E$, de Bisschop E, Vaini-Elies V, et al. Modern algorithm for treating pudendal neuralgia: 212 cases and 104 decompressions [in French]. J Gynecol Obstet Biol Reprod (Paris) 2003;32:705-12.

6. Beco J, Climov D, Bex M. Pudendal nerve decompression in perineology: A case series. BMC Surg 2004;4:15. http://dx.doi.org/10.1186/1471-2482-4-15

7. Robert R, Labat JJ, Riant $\mathrm{T}$, et al. The pudendal nerve: clinical and therapeutic morphogenesis, anatomy, and physiopathology [in French]. Neurochirurgie 2009;55:463-9. http://dx.doi.org/10.1016/i.neuchi.2009.07.004

8. Ueda $M, O$ kamoto $Y$, Ueki $M$. A pelvic retroperitoneal schwannoma arising in the right paracolpium. Gynecol Oncol 1996;60:480-3. http://dx.doi.org/10.1006/gyno.1996.0077

9. Hugues MJ, Thomas JM, Fisher C, et al. Imaging features of retroperitoneal and pelvic schwannomas. Clin Radiol 2005;60:886-93.

10. Nishi H, Kido T, Yamanishi H, et al. Endoscopic resection of a pelvic neurogenic tumor through the retroperitoneal approach. Surg Endosc 2000;14:767.

11. Nakashima J, Ueno $M$, Nakamura $K$, et al. Differential diagnosis of primary benign and malignant retroperitoneal tumors. Int J Urol 1997;4:441-6. http://dx.doi.org/10.1111/j.1442-2042.1997.tb00282.x

Correspondence: Dr. Bertrand Guillonneau, Urology Service, Surgery Department, Memorial SloanKettering Cancer Center, New York, NY; guillonb@mskcc.org 